\title{
Intolerancia a la aspirina en pacientes con poliposis nasal y asma bronquial
}

\author{
Aspirin intolerante in patients with nasal polyps and asthma
}

Pilar Gajardo 01, Ximena Fonseca $A^{2}$.

\begin{abstract}
RESUMEN
La tríada de la aspirina, caracterizada por intolerancia a la aspirina, asma bronquial y poliposis nasal, fue descrita inicialmente por Widal en 1922. La prevalencia de intolerancia a la aspirina en la población general es de aproximadamente $6 \%$, pero estudios han revelado que hasta $78 \%$ de los pacientes con poliposis nasal padecen la triada. La presentación clínica consiste en un patrón característico evolutivo de la aparición de los síntomas, presentándose en primer lugar rinitis, luego poliposis nasal, asma bronquial y finalmente IA. Si bien es sabido que esta patología corresponde a una pseudoalergia, donde ocurre una degranulación inespecífica de los mastocitos, los mecanismos fisiopatológicos que gatillan esta degranulación no están totalmente dilucidados, siendo la hipótesis más aceptada actualmente aquella que guarda relación con las modificaciones en el metabolismo del ácido araquidónico causadas por la aspirina. Actualmente no existen exámenes de laboratorio para la confirmación diagnóstica de intolerancia a la aspirina, por lo que frente a la sospecha clínica de esta patología debe someterse al paciente a pruebas de provocación para hacer el diagnóstico certero de esta enfermedad, siendo la más utilizada la prueba de provocación con aspirina oral.
\end{abstract}

Palabras clave: Intolerancia a la aspirina, poliposis nasal, triada de la aspirina.

\begin{abstract}
The aspirin triad, characterized by aspirin intolerance, bronchial asthma and nasal polyposis was first described by Widal in 1922. Prevalence of aspirin intolerance in the general population is near $6 \%$, but studies have shown that $78 \%$ of the patients with nasal polyps suffer from the triad. Clinical presentation consists of a characteristic evolutive pattern, with rhinitis appearing first, then nasal polyps, bronchial asthma and finally aspirin intolerance. Even though it is well known that this pathology corresponds to pseudoallergy, where unspecific mast cell degranulation occurs, the physiopathologic mechanisms that trigger this degranulation are not completely understood. The most accepted hypothesis involves aspirin-induced modifications in the metabolism of arachidonic acid. Currently there are no in vitro tests available for aspirin intolerance
\end{abstract}

Médico, Tesista de magíster en Ciencias Médicas, Mención Inmunología, Universidad de Chile.

2 Médico Otorrinolaringólogo, Departamento de Otorrinolaringología, Hospital Clínico de la Pontificia Universidad Católica de Chile. 
diagnosis, so if this pathology is suspected, a provocation test with aspirin is necessary to confirm the diagnosis, oral provocation test being the most frequently used in clinical practice.

Key words: Aspirin intolerance, nasal polyposis, aspirin triad.

\section{INTRODUCCIÓN}

Desde su introducción en 1897 el ácido acetil salicílico (AAS), conocido comercialmente como aspirina se ha transformado en uno de los medicamentos más utilizados en el mundo, debido a sus conocidos efectos analgésicos, antiinflamatorios e inhibitorios de la agregación plaquetaria, transformándose en la primera opción para la profilaxis de eventos cardiovasculares. En general la aspirina es bien tolerada por la mayoría de los pacientes, pero se han descrito una gama de efectos adversos relacionados con el uso de este medicamento, que van desde molestias gastrointestinales hasta la poco conocida, pero potencialmente severa intolerancia a la aspirina $(I A)^{1}$.

\section{DEFINICIONES}

Las reacciones adversas a drogas pueden ser dependientes 0 independientes de la dosis administrada. Las primeras son generalmente causadas por las propiedades farmacológicas del medicamento, son predecibles y tienen una baja mortalidad. Por el contrario, las reacciones independientes de la dosis del medicamento, son poco comunes, no se relacionan con las propiedades farmacológicas de la droga, son impredecibles y tienen una alta mortalidad ${ }^{2}$. Dentro de este último tipo de reacciones se encuentran las reacciones alérgicas, mediadas por anticuerpos tipo IgE específicos, las cuales se producen generalmente frente a la segunda exposición al fármaco, y las reacciones pseudoalérgicas, las cuales pueden ocurrir con la primera ingesta del medicamento. Estas últimas se caracterizan por una degranulación del mastocito no mediada por $\mathrm{lgE}$, sino por estímulos inespecíficos que favorecen la liberación de histamina y otros mediadores. Dentro de las reacciones pseudoalérgicas se encuentra la IA, que puede presentarse con síntomas respiratorios (rinitis, obstrucción bronquial) o cutáneos (urticaria y angioedema), siendo estos últimos la forma más frecuente de presentación ${ }^{3}$.

Se ha descrito un tipo de asma caracterizado por episodios de hiperreactividad bronquial severos secundarios a la ingesta de aspirina y otros antiinflamatorios no esteroidales (AINES), que se ha llamado asma inducido por aspirina (AIA $)^{4}$. En la literatura también existe la nomenclatura de enfermedad respiratoria exacerbada por aspirina (EREA), que engloba a los síntomas nasales y bronquiales empeorados por el consumo de éste y otros AINES.

En 1922 Widal y colaboradores fueron los primeros en describir la asociación entre poliposis nasal (PN), asma bronquial e IA. Posteriormente en 1967 Samter y Beers definieron la «tríada de la aspirina» caracterizada por intolerancia a la aspirina, asma bronquial y poliposis nasal ${ }^{5}$. Este conjunto de síntomas es también conocido como «enfermedad de la aspirina» 0 «tríada de Samter».

\section{EPIDEMIOLOGÍA}

Se ha descrito una prevalencia de IA en la población general de $0,6 \%$ a 2,5\% ${ }^{4}$. Un estudio finlandés estudió la prevalencia de IA en cuatro mil trescientos adultos utilizando un cuestionario específico. En este estudio se describe una prevalencia general de $5,7 \%$, mientras que la prevalencia de IA que se presenta con síntomas respiratorios fue de $1,2 \%{ }^{6}$. La prevalencia de AIA en la población general es de aproximadamente $0,2 \%$, pero en los pacientes con IA aumenta a $16,4 \%{ }^{3}$. Este subgrupo de pacientes representa entre el $8 \%$ y el $20 \%$ de pacientes asmáticos adultos ${ }^{7}$. Tanto la IA como el AIA son más frecuentes en mujeres, con una relación de 2,5:1. 
Entre $1 \%$ y $4 \%$ de la población general presenta PN, la prevalencia aumenta a $16,5 \%$ en el subgrupo de pacientes con $a s m a^{6}$ y a $60 \%$ en los pacientes con $I A^{8}$.

Finalmente, la prevalencia de la tríada de la aspirina en la población general es de aproximadamente $2 \%^{3}$. De los pacientes con IA $78 \%$ tiene la tríada ${ }^{1}$. Entre $48 \%$ y $95 \%$ de los pacientes con AIA presentan $\mathrm{PN}^{9}$ y de los pacientes con PN hasta el $70,8 \%$ tiene IA y asma bronquial ${ }^{10}$.

\section{PRESENTACIÓN CLÍNICA}

La tríada de la aspirina se presenta clínicamente como una enfermedad evolutiva, con un patrón característico en la aparición de los síntomas. El cuadro clínico se inicia típicamente entre los treinta y cuarenta años de edad, con síntomas de rinitis persistente, los cuales aparecen generalmente luego de una infección viral. Alrededor de dos años después de iniciada la rinitis aparece la PN, el asma puede desarrollarse dentro de tres meses a cinco años de iniciada la rinitis y finalmente los síntomas respiratorios inducidos por aspirina pueden aparecer a lo largo de todo el curso de la enfermedad ${ }^{11}$. Este cuadro clínico no sólo es más frecuente en mujeres, sino que en ellas se presenta más precozmente y evoluciona con mayor agresividad.

Una típica reacción nasal inducida por aspirina ocurre dentro de treinta minutos a dos horas de ingerido el medicamento. Sus síntomas clásicos son rinorrea serosa y congestión nasal. Al contrario de la rinitis alérgica, edema y estornudos no son predominantes ${ }^{5}$.

Con respecto al AIA se ha descrito que $50 \%$ de los pacientes sufre de un asma severa, y requieren de corticoides sistémicos para el control de su enfermedad $^{12}$. Esta severidad puede estar asociada a la mayor prevalencia de PN que presentan estos pacientes, ya que esta enfermedad produce una mayor obstrucción de la vía aérea, o a factores solubles que afectan tanto la mucosa nasal como la mucosa bronquial.

La influencia de atopía en el desarrollo de la enfermedad se estudió en un trabajo multicéntrico realizado en Europa el cual reveló que un tercio de Ios pacientes con AIA presentan atopía. Este subgrupo de pacientes atópicos presenta síntomas de rinitis y asma bronquial más precozmente que los pacientes no atópicos, no obstante, el diagnóstico de IA y PN fue a edades similares que los pacientes no atópicos ${ }^{7}$.

Histológicamente la vía aérea de pacientes con AIA muestra signos de inflamación persistente similares a los presentes en todos los pacientes asmáticos, sin embargo, en biopsias bronquiales de pacientes con AIA los eosinófilos son cuatro veces más numerosos que en pacientes con asma tolerante a la aspirina, y quince veces más numerosos que en biopsias de mucosa normal ${ }^{13}$.

Los pacientes portadores de la tríada representan el $7 \%$ de los casos de rinosinusitis crónica ${ }^{14}$. La PN en los pacientes con IA se caracteriza por tener un comportamiento agresivo, ocupando casi la totalidad de las fosas nasales ${ }^{5}$. La extensión de la rinosinusitis es mayor en los pacientes con IA en comparación con aquellos tolerantes a la aspirina que presentan un cuadro clínico de igual severidad $^{10}$.

El aspecto macroscópico de los pólipos en los pacientes con la tríada es similar al de otros tipos de $\mathrm{PN}^{5}$. A nivel microscópico, los cilios de la mucosa nasal son normales desde un punto de vista estructural y funcional. No se ha observado ningún efecto de la aspirina en la función de los cilios de los pacientes con la tríada de la aspirina ${ }^{15}$.

Con respecto a sujetos normales, pacientes con rinitis sensible a la aspirina tienen un mayor número de eosinófilos totales y activados, mayor número de mastocitos, linfocitos $\mathrm{T}$ activados y macrófagos. Adicionalmente, en estos pacientes se ha visto un mayor número de células que expresan ARN mensajero de interleuquina 5 (IL-5), una citoquina importante en la quimiotaxis de eosinófilos ${ }^{16,17}$.

\section{FISIOPATOLOGÍA}

Los mecanismos fisiopatológicos de la IA y los síntomas respiratorios asociados a ella no están totalmente dilucidados. La hipótesis más aceptada actualmente tiene relación con las modificaciones en el metabolismo del ácido araquidónico (AA) causadas por la aspirina y otros AINES. 
El $A A$ es un ácido graso esencial que se encuentra esterificado en los fosfolípidos de la membrana plasmática celular de eosinófilos y mastocitos. Existen dos vías de metabolización del AA, la vía de la cicloxigenasa (COX) y la vía de la lipoxigenasa (LOX), las cuales dan origen a distintos eicosanoides (Figura 1). La enzima COX existe en dos isoformas, COX-1 que se expresa de manera constitutiva, y COX-2 la cual es inducible en condiciones inflamatorias. Bajo condiciones fisiológicas los metabolitos producidos por la vía de COX son prostaglandina E2 (PGE2), la cual cumple un importante rol en la inhibición de la liberación de cisteinil leucotrienos (cys-L) e inhibe la broncoconstricción, prostaglandina 12 (prostaciclina) y tromboxanos. En condiciones inflamatorias también se produce prostaglandina F2 y prostaglandina D2 (PGD2), la cual produce vasodilatación, broncoconstricción y es un potente atrayente para eosinófilos ${ }^{1}$.

Mediante la vía de la LOX el AA se convierte en leucotrieno A4 (LTA4), el cual puede ser hidrolizado, dando origen al leucotrieno B4 (LTB4), el cual atrae y activa a neutrófilos. El LTA4 puede también ser convertido en cys-L (LTC4, LTD4 y LTE4) mediante la enzima LTC4 sintasa (LTC4S). Los cys-L aumentan la permeabilidad vascular, favorecen la exudación del plasma y formación de edema, atraen eosinófilos, estimulan la hiperproducción de mucus por células bronquiales y estimulan la broncoconstricción.

Una de las teorías con respecto a la alteración del metabolismo del AA en pacientes con la tríada de la aspirina plantea que la aspirina y otros AINES inhibirían a la enzima COX-1, inclinando el metabolismo del AA hacia la vía de LOX, con el consiguiente aumento de los niveles de cys- $L$ y por ende sus acciones perjudiciales a nivel nasal y bronquial'. Con respecto a esta teoría existe un estudio que muestra que los pacientes asmáticos tolerantes e intolerantes a la aspirina presentan niveles basales elevados de cys-L, en comparación con sujetos sanos, y que luego de un estímulo bronquial con aspirina, los pacientes intolerantes a la aspirina muestran un aumento aún mayor de los niveles de cys-L. Este mismo estudio muestra que los pacien-

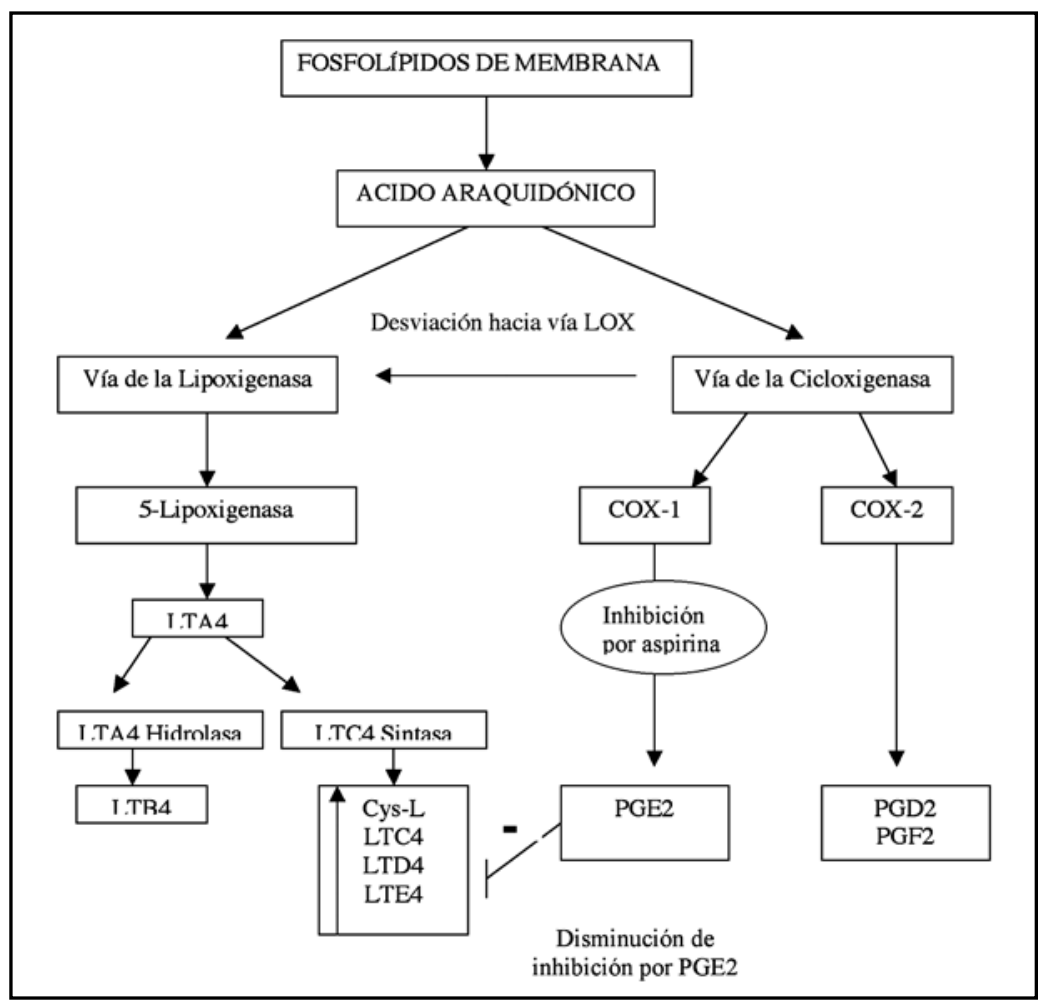

Figura 1. Metabolismo del ácido araquidónico y modificaciones causadas por la aspirina. 
tes con AIA tienen niveles basales disminuidos de PGE2 ${ }^{18}$.

Los pacientes con AIA presentan niveles elevados de cys- $L$ en orina, los cuales se asocian con la presencia de PN y rinosinusitis crónica, y disminuyen luego de cirugía sinusal, sugiriendo que sería la mucosa nasosinusal la fuente principal de cys-L en estos pacientes ${ }^{19}$.

Se ha observado que los pacientes con AIA presentan un aumento en el número de células que expresan la enzima LTC4S en biopsias bronquiales. La expresión de esta enzima en pacientes con AlA es cinco veces mayor que en pacientes con asma tolerante a la aspirina y dieciocho veces mayor en comparación con sujetos normales ${ }^{20}$. Estudios recientes también muestran un aumento de expresión de LTC4S en eosinófilos presentes en la mucosa de pólipos nasales de pacientes con AIA ${ }^{21}$.

Finalmente un estudio realizado en Inglaterra mostró que en los pacientes con rinosinusitis crónica e IA las células inflamatorias de la submucosa nasal tienen una mayor expresión selectiva del receptor de cys-L tipo 1(RCysLT1), la cual disminuye con el tratamiento de desensibilización con aspirina. Los autores proponen que junto con el aumento de cys-L, la mayor expresión de este receptor jugaría un rol importante en la fisiopatología de la enfermedad $^{22}$.

Con respecto a la búsqueda de marcadores genéticos de esta enfermedad, se ha descrito una asociación entre el alelo HLA-DPB1 0301 y el fenotipo de AIA en individuos coreanos y pola$\cos ^{1}$. También se han descrito polimorfismos de un único nucleótido en genes que codifican para enzimas y receptores relacionados con el metabolismo del AA en pacientes con AIA, como es el caso de la enzima $\mathrm{LTC}_{\mathrm{S}} \mathrm{S}^{23}$ y el receptor de cys-L tipo 2.

\section{DIAGNÓSTICO}

Actualmente no existen exámenes de laboratorio para la confirmación diagnóstica de IA, por lo que frente a la sospecha clínica de esta patología debe someterse al paciente a pruebas de provocación con aspirina para hacer el diagnóstico certero de esta enfermedad ${ }^{11}$, las cuales consisten en administrar dosis crecientes de aspirina y evaluar la presencia de síntomas en relación a la ingesta del medicamento. Existen cuatro vías de provocación con aspirina: oral, bronquial, nasal y endovenosa, esta última no es usada regularmente. Los médicos encargados de llevar a cabo esta prueba diagnóstica deben estar preparados para tratar agresivamente las reacciones adversas a la aspirina, ya que los pacientes pueden presentar broncoespasmos severos e incluso efectos vasculares, con el potencial riesgo de shock ${ }^{24}$.

Para poder someterse al test de provocación, el paciente debe tener un $\mathrm{VEF}_{1}$ igual o mayor a $70 \%{ }^{25}$. Las contraindicaciones para la realización del test de provocación con aspirina se describen en la Tabla 1. Es importante señalar que los pacientes deben continuar el tratamiento con corticoides (orales y/o tópicos) al momento del diagnóstico, ya que suspender estos medicamentos puede empeorar la patología de base del paciente con la subsiguiente declinación de su función pulmonar, lo cual puede ser un impedimento para llevar a cabo la prueba diagnóstica. Sin embargo, hay algunos medicamentos que deben suspenderse ${ }^{25}$ :

- Suspender ocho horas antes: cromolina, broncodilatadores de corta acción y bromuro de ipatropio.

- Suspender cuarenta y ocho horas antes: broncodilatadores de larga acción, teofilina y bromuto de tiotropio.

- Suspender tres días antes: antihistamínicos de corta acción.

Tabla 1. Contraindicaciones para la realización del test de provocación oral y bronquial con aspirina ${ }^{25}$
1. Historia de anafilaxis severa causada por aspirina u otros AINES (considerar provocación nasal).
2. Enfermedad severa cardiaca, digestiva, hepática y/o renal.
3. Infección del tracto respiratorio cuatro semanas previo a la realización del test.
4. Embarazo.
5. Tratamiento actual con betabloqueadores. 
- Suspender una semana antes: modificadores de la acción de leucotrienos.

El test de provocación oral (TPO) es considerado gold standard para el diagnóstico de reacciones respiratorias y cutáneas causadas por la aspirina'. Se han descrito distintos protocolos de este tipo de prueba diagnóstica, los cuales difieren en la dosis de aspirina administrada, los intervalos entre cada dosis y los criterios de positividad del test ${ }^{26}$. El más utilizado es un protocolo de tres días en el cual durante el primer día se realiza una espirometría y se fija el valor basal de $\mathrm{VEF}_{1}$, posteriormente se administran tres dosis de placebo, con un intervalo de dos horas entre ellas. Se realiza una espirometría cada treinta minutos, para evaluar la estabilidad de la vía aérea, ya que si el VEF, varía más del $15 \%$ el paciente no puede llevar a cabo el TPO. En el segundo día se comienza con la administración de dosis crecientes de aspirina, con intervalos de dos horas, hasta alcanzar una dosis acumulativa de 500 mg de aspirina ( $10 \mathrm{mg}, 27 \mathrm{mg}, 44 \mathrm{mg}, 117 \mathrm{mg}, 312$ $\mathrm{mg}, 500 \mathrm{mg}$ ). Antes de cada dosis se realiza una espirometría, la cual se repite cada treinta minutos durante el estudio. Además se evalúa la aparición de síntomas respiratorios como broncoespasmo, rinorrea, congestión nasal y síntomas extrarrespiratorios como inyección ocular, eritema y náuseas. Los criterios de positividad son ${ }^{25}$.

- Disminución del VEF Vayor $_{1}$ igual al 20\%.

- Aparición de síntomas extrabronquiales severos, como rinorrea profusa, aunque el $\mathrm{VEF}_{1}$ no sea menor al $20 \%$.

Otro método utilizado para la confirmación de IA es la prueba de provocación bronquial 0 inhalatoria (TPB) con acetilsalicilato de lisina (alis), que es una sal soluble derivada del ácido acetilsalicílico (AAS) que se puede emplear por vía oral y parenteral, quedando libre en el organismo posteriormente el AAS. $1.000 \mathrm{mg}$ de a-lis equivalen a $500 \mathrm{mg}$ de AAS.

Nizankowska ${ }^{26}$ y cols compararon la eficacia diagnóstica de la provocación oral versus provocación bronquial y observaron que ambos test tienen especificidades similares, pero el TPO mostró una sensibilidad mayor, aunque esta diferencia no fue estadísticamente significativa. La mayor ventaja del TPB fue el menor tiempo requerido para realizarlo. La inclusión de síntomas extrabronquiales aumentó el valor diagnóstico de ambas pruebas.

El test de provocación nasal (TPN) con a-lis ha demostrado ser un test simple, seguro y rápido en el diagnóstico de AIA e IA caracterizada principalmente por síntomas nasales ${ }^{27}$; no obstante, el TPN ha demostrado tener algunas limitaciones, ya que en aquellos pacientes con una función nasal alterada 0 aquellos que presentan una hiperreactividad nasal inespecífica, la respuesta no sería valorable ${ }^{28}$. Recientemente se ha implementado un TPN utilizando una solución de ketorolaco diluido ${ }^{29}$, siendo una alternativa al TPN con a-lis en Estados Unidos, debido a que esta última no ha sido aprobada para su uso en humanos en ese país.

Micheletto y $\operatorname{cols}^{30}$ relacionaron la respuesta nasal y los niveles de leucotrienos en orina luego de TPN con a-lis en pacientes asmáticos tolerantes e intolerantes a la aspirina, observando que sólo los pacientes con AIA presentaron respuestas nasales positivas (medidas mediante rinometría acústica) y aumento de leucotrienos urinarios.

EI TPN puede utilizarse como estudio inicial en el diagnóstico de IA. Pacientes con alta sospecha de IA con un resultado negativo en el TPN deberían someterse a TPB o TPO con aspirina para confirmar su diagnóstico. En la Tabla 2 se encuentra una

Tabla 2. Comparación del rendimiento de las distintas pruebas diagnósticas para la intolerancia a la aspirina ${ }^{25}$

\begin{tabular}{|lcc|}
\hline Test & Sensibilidad & Especificidad \\
\hline TP0 aspirina & $89 \%$ & $93 \%$ \\
TPB aspirina & $77 \%$ & $93 \%$ \\
TPB a-lis & $90 \%$ & $90 \%$ \\
TPN a-lis & $80 \%-86,7 \%$ & $92,5 \%-95,7 \%$ \\
\hline
\end{tabular}


comparación de sensibilidad y especificidad de los distintos tipos de test de provocación con aspirina.

Es importante señalar que todas las pruebas de provocación deben ser precedidas por una provocación con placebo, para excluir la variabilidad de la respuesta naso-bronquial ${ }^{13}$.

\section{TRATAMIENTO}

\section{Tratamiento de la(s) patología(s) de base}

Como se dijo anteriormente, los pacientes con la tríada de la aspirina sufren de un asma severa, por lo cual el tratamiento eficaz de su patología debe incluir el tratamiento adecuado del asma bronquial, lo cual en la mayoría de los casos incluye el uso de corticoides sistémicos. Un estudio que incluyó trescientos pacientes con EREA observó que el $77 \%$ de ellos era usuario de corticoides sistémicos, $22 \%$ lo hacía de manera diaria, con una dosis promedio de prednisona de $7,5 \mathrm{mg}$ al día ${ }^{31}$. Con respecto al uso de modificadores de la acción de leucotrienos, existen publicaciones que muestran el beneficio de estas drogas en el tratamiento del AIA. Un estudio mostró que al agregar el inhibidor de la enzima 5-lipoxigenasa, zileuton, en una dosis de $600 \mathrm{mg}$ cuatro veces al día, al tratamiento de pacientes con AIA, existe una mejoría clínica de estos pacientes. El uso de este medicamento también disminuyó los niveles de leucotrienos urinarios en pacientes con $\mathrm{AlA}^{32}$. Un estudio de los mismos autores, evaluó los beneficios de agregar un antagonista de leucotrienos, montelukast, al tratamiento de pacientes con AIA. El grupo que recibió montelukast mostró una mejoría en los síntomas asmáticos, en la función pulmonar y en la calidad de vida en comparación con los pacientes que recibieron placebo ${ }^{33}$. Con respecto a los beneficios que ofrece el tratamiento con montelukast a los pacientes con PN e IA, diversos estudios han demostrado que el uso de este medicamento se asocia con una menor recurrencia de PN poscirugía ${ }^{34}$ y con una mejoría en la calidad de vida de los pacientes ${ }^{35}$, en comparación con el uso de placebo. Estos resultados sugieren que el agregar modificadores de la acción de los leucotrienos mejoraría el tratamiento en los pacientes con la tríada de la aspirina y serían una alternativa terapéutica que permitiría disminuir las dosis de corticoides sistémicos utilizados en estos pacientes, disminuyendo así los potenciales efectos adversos de los glucocorticoides.

\section{Prevención y seguridad en el consumo de otros AINES}

Una vez hecho el diagnóstico de tríada de la aspirina el paciente debe ser educado acerca de las posibles reacciones cruzadas entre aspirina y otros AINES, siendo de gran utilidad la entrega de una lista con los nombres de medicamentos que el paciente no puede consumir. Se ha descrito una reactividad cruzada del $100 \%$ entre aspirina y los AINES que inhiben COX-1, por lo cual estos últimos están contraindicados en pacientes con la tríada ${ }^{11}$. Con respecto al uso del paracetamol, inhibidor parcial de COX-1, está descrito que la mayoría de los pacientes tolera adecuadamente $500 \mathrm{mg}$ de esta droga, hasta $28 \%$ tiene reacciones respiratorias leves con $1 \mathrm{gr}$ y $6 \%$ adicional reaccionó a $1,5 \mathrm{gr}$ de paracetamol ${ }^{11}$. Actualmente están disponibles en el mercado AINES que inhiben selectivamente a la enzima COX-2, Ios cuales inicialmente se consideraban de uso seguro en pacientes con IA. Estudios recientes ${ }^{36}$ han demostrado que $7 \%$ y $3 \%$ de pacientes con IA presentan síntomas respiratorios frente a la ingesta de dosis habituales de nimesulide y meloxicam, respectivamente, por lo cual estos medicamentos ya no son de uso aconsejable en pacientes con IA. Los AINES más seguros son celecoxib y refecoxib ${ }^{37,38}$. Sin embargo, antes de permitir el uso de AINES en un paciente con IA se recomienda realizar un TPO para el medicamento particular, certificando de esta manera su uso seguro para el paciente.

Los pacientes con la tríada de la aspirina pueden someterse a cirugía para la resolución quirúrgica de los PN. Es importante recalcar la importancia de la evaluación por el anestesista, previo a la cirugía, debido a que éstos pueden presentar crisis asmáticas severas durante el período posoperatorio debido a su disfunción pulmonar. Otra tarea del anestesista es planear, en conjunto con el paciente, el uso adecuado de analgésicos poscirugía ${ }^{39}$.

Con respecto a la mejoría posquirúrgica de los síntomas en pacientes con PN e IA existen resultados contradictorios. Algunos autores han encon- 
trado que los pacientes con la tríada de la aspirina obtienen menores beneficios posquirúrgicos en comparación con pacientes tolerantes a la aspiri$\mathrm{na}^{40}$, sin embargo, estudios posteriores relatan igual mejoría sintomática y en la calidad de vida en ambos subgrupos luego de cirugía endoscópica ${ }^{41}$. Se ha visto que la cirugía endoscópica destinada a corregir los pólipos nasales, mejora también el componente asmático de los pacientes con la tríada. Esta mejoría en los síntomas asmáticos es mayor al año poscirugía y se mantiene hasta un período de diez años ${ }^{42}$.

\section{Desensibilización}

Existen pacientes con la tríada de la aspirina que cumplen los criterios para someterse a una terapia de desensibilización (Tabla 3). Estos criterios son bastante estrictos ya que este tratamiento consiste en exponer al paciente a dosis crecientes del fármaco con el fin de inducir tolerancia inmunológica, lo cual tiene algunos efectos adversos como, por ejemplo, sangramiento gastrointestinal. Los mecanismos fisiopatológicos de la desensibilización no están totalmente establecidos, pero se sabe que este tratamiento disminuye la expresión de RCysLT1, disminuye la producción de tromboxano B2 y LTB4 en monocitos, inhibe la liberación de histamina y PGD2 por mastocitos ${ }^{43}$, y posiblemente influye en los mecanismos de señalización intracelular de la vía de los cys-L ${ }^{14}$. Desde un punto de vista clínico los pacientes sometidos a desensibilización muestran no sólo una mejoría sintomática, sino que también logran disminuir la dosis de corticoides necesarias para un adecuado control de la enfermedad ${ }^{44}$. A pesar del éxito reportado, esta terapia permanece subutilizada en el enfrentamiento de estos pacientes.

Hasta la fecha se han descrito distintos protocolos de desensibilización, que varían en la vía de administración y dosis de aspirina.

La desensibilización oral fue la primera descrita y es actualmente la más utilizada. Puede llevarse a cabo bajo un protocolo extrahospitalario de dos a tres días si el paciente y el centro de atención cumplen con los requisitos necesarios, los cuales se describen en la Tabla 4, y que tienen relación con el monitoreo constante del paciente, ya que el principal riesgo de esta terapia es la aparición de síntomas respiratorios graves e incluso shock.

Este protocolo se inicia con una evaluación que tiene como objetivo constatar que el paciente se encuentra estable de su patología de base, con el fin de disminuir los efectos adversos de esta

Tabla 3. Criterios de inclusión para desensibilización con aspirina ${ }^{50}$

1. Enfermedad vascular con indicación de antiagregación plaquetaria con inhibidores de COX-1 a permanencia.

2. Asma bronquial o rinosinusitis con respuesta clínica subóptima a tratamiento con corticoides sistémicos y modificadores de la acción de leucotrienos.

3. Poliposis nasal recurrente, que ha requerido múltiples polipectomías.

Tabla 4. Criterios de terapia intrahospitalaria y ambulatoria para desensibilización oral con aspirina ${ }^{50}$

\begin{tabular}{|ll|}
\hline Terapia Intrahospitalaria & Terapia Ambulatoria \\
\hline Uso betabloqueadores & Médico disponible siempre para evaluar al paciente y manejar eventua- \\
& les complicaciones \\
Infarto agudo al miocardio reciente & $\begin{array}{l}\text { Personal de salud experimentado para evaluación y atención al pacien- } \\
\text { te y eventuales complicaciones }\end{array}$ \\
Asma severa & $\begin{array}{l}\text { Un miembro del equipo médico con dedicación exclusiva al paciente } \\
\text { durante toda la desensibilización }\end{array}$ \\
Antecedente shock anafiláctico por & $\begin{array}{l}\text { Equipo de monitorización cardiopulmonar disponible durante toda la } \\
\text { aspirina u otros AINES }\end{array}$ \\
\hline
\end{tabular}


terapia. En la Tabla 5 se enumeran los pasos de esta evaluación inicial, la cual debe realizarse entre uno a siete días antes del tratamiento de desensibilización con aspirina.

Si el paciente se encuentra estable se programa el inicio de la desensibilización. El protocolo se inicia con la administración de $30 \mathrm{mg}$ de aspirina, y luego cada tres horas se administran consecutivamente dosis crecientes del fármaco $(30 \mathrm{mg}, 45$ $\mathrm{mg}, 60 \mathrm{mg}, 100 \mathrm{mg}, 150 \mathrm{mg}, 325 \mathrm{mg}$ y $650 \mathrm{mg}$ ). La menor dosis de aspirina a la cual el paciente presenta síntomas se denomina dosis de provocación (habitualmente entre $30 \mathrm{mg}$ y $100 \mathrm{mg}$ ). Debe realizarse una evaluación clínica y medición de $V_{E F}{ }_{1}$ cada una hora, teniendo presente de tratar adecuadamente los síntomas del paciente, acorde con la naturaleza de éstos, y luego de que el paciente esté asintomático proseguir con la dosis siguiente. Si ocurren síntomas nasales, oculares 0 gastrointestinales durante el primer día, debe tratarse al paciente con antihistamínicos a lo largo de todo el protocolo. Luego de establecer la dosis de provocación ésta debe repetirse, lo cual puede llevarse a cabo el primer día, o si no se cuenta con el tiempo necesario, se inicia el segundo día y posteriormente se continúa con dosis crecientes del fármaco hasta lograr que el paciente permanezca asintomático con $650 \mathrm{mg}$ de aspirina, con lo cual se considera desensibilizado. Posteriormente el paciente debe continuar con una dosis de mantención, ya que si el paciente desensibilizado no consume aspirina por un plazo igual o mayor a cuarenta y ocho horas, se considera nuevamente susceptible a la aspirina. Un estudio reciente recomienda iniciar una dosis de mantención de $650 \mathrm{mg}$ dos veces al día y si el paciente permanece estable por un mes se puede disminuir la dosis de mantención progresivamente, hasta la mínima dosis efectiva (generalmente $325 \mathrm{mg}$ una vez al día) ${ }^{45}$.
Buchimiller y cols ${ }^{50}$ han desarrollado un nuevo protocolo de desensibilización para pacientes con PN e IA refractaria a tratamiento, en el cual dosis crecientes de aspirina son administradas con intervalos de una hora, con el fin de acortar la duración del protocolo, teniendo hasta la fecha un éxito del $47 \%$ en lograr la desensibilización en un solo día, y $100 \%$ en dos días (versus tres días del protocolo habitual).

En los pacientes IA con enfermedad cardiovascular que necesitan la implantación urgente de un stent coronario y mantención de tratamiento antiplaquetario, se ha establecido un protocolo de desensibilización rápida de aproximadamente tres horas de duración. Este protocolo se inicia con 1 mg de aspirina, doblando la dosis cada treinta minutos hasta alcanzar $100 \mathrm{mg}$ del fármaco, logrando excelentes resultados. La dosis de mantención de estos pacientes es menor, generalmente entre 75 y $100 \mathrm{mg}$, lo que se ajusta a su tratamiento profiláctico $0^{46}$. Un estudio realizado en Italia relata que de mil catorce pacientes ingresados para cateterización coronaria, veintiséis $(2,6 \%)$ tenían historia de IA, y de éstos diez presentaban AIA. De los diez pacientes con AIA todos fueron sometidos a un esquema de desensibilización rápida, que consistió en la administración de dosis crecientes de aspirina (1 mg, $5 \mathrm{mg}, 10 \mathrm{mg}, 20 \mathrm{mg}, 40 \mathrm{mg} \mathrm{y}$ $100 \mathrm{mg}$ ), las cuales fueron ingeridas con un intervalo de treinta a ciento veinte minutos, teniendo el protocolo de desensibilización una duración total de cinco horas y media. De los diez pacientes, nueve tuvieron una desensibilización exitosa $(90 \%)$, y de éstos solamente un paciente había suspendido el uso de aspirina al año de seguimiento, debido a una úlcera péptica ${ }^{47}$.

Se ha descrito un protocolo de desensibilización por vía endonasal, el cual está indicado en pacientes cuyos síntomas son preferentemente

\section{Tabla 5. Determinación de la estabilidad de la vía aérea previo al inicio de la desensibilización con aspirina ${ }^{50}$}

1. $\mathrm{VEF}_{1}>70 \%$ del valor esperado (valor absoluto $>1,5 \mathrm{~L}$ ).

2. Medición de $\mathrm{VEF}_{1}$ cada tres horas: menos de $10 \%$ de variabilidad.

3. Iniciar o continuar montelukast, $10 \mathrm{mg} /$ día.

4. Iniciar o continuar corticoides inhalatorios/broncodilatadores de acción prolongada.

5. Dar pulso de corticoides sistémicos en caso de bajo $\mathrm{VEF}_{1} 0$ inestabilidad bronquial.

6. Descontinuar antihistamínicos cuarenta y ocho horas antes del tratamiento con aspirina. 
nasales. Se lleva a cabo administrando dosis de alis equivalentes a 20, 200 y 2.000 microgramos de aspirina, existiendo resultados contradictorios con respecto a su utilidad, ya que si bien se ha mostrado beneficios en el tratamiento de la PN en pacientes con $I A^{43}$, un estudio reciente no mostró beneficios clínicos de esta vía de desensibilización, pero evidenció que las células inflamatorias de la submucosa nasal de pacientes sometidos a desensibilización nasal disminuyeron la expresión del RCysLT1. Este cambio a nivel microscópico sugiere que este tipo de desensibilización tiene efectos moleculares, y que el hecho de no encontrar beneficio clínico podría deberse a que el tamaño del grupo de estudio fue muy pequeño (once pacientes).

Un grupo estadounidense ha descrito un protocolo mixto de provocación y desensibilización que consiste en la administración de cinco dosis crecientes de ketorolaco por vía endonasal, con un intervalo de treinta minutos entre cada dosis. Posterior a la desensibilización intranasal los pacientes reciben una dosis de $60 \mathrm{mg}$ de aspirina, la cual es repetida a los noventa minutos. A la mañana siguiente se administra una dosis de aspirina de $150 \mathrm{mg}$ y tres horas después una última dosis de $325 \mathrm{mg}$ de aspirina. De diez pacientes sometidos a este protocolo nueve fueron desensibilizados satisfactoriamente, lo cual demuestra la eficacia, seguridad y eficiencia de este nuevo enfoque diagnóstico-terapéutico49.

\section{CONCLUSIONES}

La tríada de la aspirina es una patología poco frecuente, pero el otorrinolaringólogo en su práctica clínica puede verse enfrentado con una frecuencia mayor de lo sospechada a pacientes en los cuales la intolerancia a la aspirina esté jugando un rol importante en la sintomatología y gravedad de la patología nasal y respiratoria. Actualmente el diagnóstico de esta enfermedad se basa en las pruebas de provocación. Una vez hecho el diagnóstico debe tenerse en cuenta las variaciones clínicas de esta enfermedad e instruir al paciente acerca de los AINES que puede consumir y si la clínica del paciente lo requiere puede someterse a terapia de desensibilización con aspirina, la cual ha demostrado tener excelentes resultados en disminuir los síntomas de estos pacientes.

\section{BIBLIOGRAFÍA}

1. Jenneck $C$, Juergens $U$, Buecheler $M$, and Novak N. Pathogenesis, diagnosis, and treatment of aspirin intolerance. Ann Allergy Asthma Immunol 2007; 99: 13-21.

2. Edwards IR AND ARonson JK. Adverse drug reactions: definitions, diagnosis, and management. Lancet 2000; 356: 1255-9.

3. Schiavino D, Nucera E, Milani A, et al. The aspirin disease. Thorax 2000; 55 Suppl 2: S66-9.

4. Slepian IK, Mathews KP, and McLean JA. Aspirinsensitive asthma. Chest 1985; 87: 386-91.

5. PICAdo C. Aspirin intolerance and nasal polyposis. Curr Allergy Asthma Rep 2002; 2: 488-93.

6. Hedman J, Kaprio J, Poussa T, and Nieminen MM. Prevalence of asthma, aspirin intolerance, nasal polyposis and chronic obstructive pulmonary disease in a population-based study. Int J Epidemiol 1999; 28: 717-22.

7. Szczeklik A, Nizankowska E, and Duplaga M. Natural history of aspirin-induced asthma. AIANE Investigators. European Network on AspirinInduced Asthma. Eur Respir J 2000; 16: 432-6.

8. Pawankar R. Nasal polyposis: an update: editorial review. Curr Opin Allergy Clin Immunol 2003; 3: 1-6.

9. Ceylan E, Gencer M, and San I. Nasal polyps and the severity of asthma. Respirology 2007; 12 : 272-6.

10. KowALSKI ML. Rhinosinusitis and nasal polyposis in aspirin sensitive and aspirin tolerant patients: are they different? Thorax 2000; 55 Suppl 2: S84-6.

11. Stevenson DD and Szczeklik A. Clinical and pathologic perspectives on aspirin sensitivity and asthma. J Allergy Clin Immunol 2006; 118: 773-86; quiz 787-8.

12. PfaAr 0 and Klimek L. Eicosanoids, aspirinintolerance and the upper airways-current standards and recent improvements of the desensitization therapy. J Physiol Pharmacol 2006; 57 Suppl 12: 5-13.

13. Szczeklik A and Stevenson DD. Aspirin-induced asthma: advances in pathogenesis, diagnosis, 
and management. J Allergy Clin Immunol 2003; 111: 913-21; quiz 922.

14. Hosemann W. Surgical treatment of nasal polyposis in patients with aspirin intolerance. Thorax 2000; 55 Suppl 2: S87-90.

15. Lewis FH, Beals TF, Carey TE, Baker SR, and Mathews KP. Ultrastructural and functional studies of cilia from patients with asthma, aspirin intolerance, and nasal polyps. Chest 1983; 83: 487-90.

16. Varga eM, Jacobson MR, Masuyama K, et al. Inflammatory cell populations and cytokine mRNA expression in the nasal mucosa in aspirin-sensitive rhinitis. Eur Respir J 1999; 14: 610-5.

17. Park HS, Nahm DH, Park K, Suh KS, and Yim HE. Immunohistochemical characterization of cellular infiltrate in nasal polyp from aspirinsensitive asthmatic patients. Ann Allergy Asthma Immunol 1998; 81: 219-24.

18. Schafer D, Schmid M, Gode UC, and Baenkler HW. Dynamics of eicosanoids in peripheral blood cells during bronchial provocation in aspirinintolerant asthmatics. Eur Respir J 1999; 13: 638-46.

19. Higashi N, Taniguchi M, Mita H, et al. Clinical features of asthmatic patients with increased urinary leukotriene E4 excretion (hyperleukotrienuria): Involvement of chronic hyperplastic rhinosinusitis with nasal polyposis. J Allergy Clin Immunol 2004; 113: 277-83.

20. Cowburn AS, Sladek K, Soja J, et al. Overexpression of leukotriene C4 synthase in bronchial biopsies from patients with aspirinintolerant asthma. J Clin Invest 1998; 101: 834-46.

21. Adamjee J, Suh YJ, Park HS, et al. Expression of 5-lipoxygenase and cyclooxygenase pathway enzymes in nasal polyps of patients with aspirin-intolerant asthma. J Pathol 2006; 209: 392-9.

22. Sousa AR, Parikh A, Scadding G, Corrigan CJ, and LEE TH. Leukotriene-receptor expression on nasal mucosal inflammatory cells in aspirinsensitive rhinosinusitis. N Engl J Med 2002; 347: 1493-9.

23. Sanak M, Pierzchalska M, Bazan-Socha S, and Szczexlik A. Enhanced expression of the leukotriene $C(4)$ synthase due to overactive transcription of an allelic variant associated with aspirin-intolerant asthma. Am J Respir Cell Mol Biol 2000; 23: 290-6.

24. Namazy JA and Simon RA. Sensitivity to nonsteroidal anti-inflammatory drugs. Ann Allergy Asthma Immunol 2002; 89: 542-50; quiz 550, 605.

25. Nizankowska-Mogilnicka E, Bochenek $G$, Mastalerz L, ET AL. EAACI/GA2LEN guideline: aspirin provocation tests for diagnosis of aspirin hypersensitivity. Allergy 2007; 62: 1111-8.

26. Nizankowska E, Bestynska-Krypel $A$, Cmiel $A$, and SzCZEKLIK A. Oral and bronchial provocation tests with aspirin for diagnosis of aspirininduced asthma. Eur Respir J 2000; 15: 863-9.

27. Milewski M, Mastalerz L, Nizankowska E, and SzCZEKLIK A. Nasal provocation test with lysineaspirin for diagnosis of aspirin-sensitive asthma. J Allergy Clin Immunol 1998; 101: 581-6.

28. Alonso A, and Vives R. Test de provocación bronquial y nasal con acetil salicilato de lisina en el diagnóstico de intolerancia a AINES. Alergol Imunol Clin 2002; 17: 168-174.

29. White A, Bigby T, and Stevenson D. Intranasal ketorolac challenge for the diagnosis of aspirinexacerbated respiratory disease. Ann Allergy Asthma Immunol 2006; 97: 190-5.

30. Micheletto C, Tognella S, Visconti M, Trevisan F, and DaL Negro RW. Changes in urinary LTE4 and nasal functions following nasal provocation test with ASA in ASA-tolerant and -intolerant asthmatics. Respir Med 2006; 100: 2144-50.

31. Berges-Gimeno MP, Simon RA, and Stevenson DD. The natural history and clinical characteristics of aspirin-exacerbated respiratory disease. Ann Allergy Asthma Immunol 2002; 89: 474-8.

32. Dahlen B, Nizankowska E, Szczeklik A, et al. Benefits from adding the 5-lipoxygenase inhibitor zileuton to conventional therapy in aspirin-intolerant asthmatics. Am J Respir Crit Care Med 1998; 157: 1187-94.

33. Dahlen Se, Malmstrom K, Nizankowska e, et al. Improvement of aspirin-intolerant asthma by montelukast, a leukotriene antagonist: a randomized, double-blind, placebo-controlled trial. Am J Respir Crit Care Med 2002; 165: 9-14.

34. Grundmann T and Topfner M. [Treatment of ASSAssociated Polyposis (ASSAP) with a cysteinyl 
leukotriene receptor antagonist - a prospective drug study on its antiinflammatory effects]. Laryngorhinootologie 2001; 80: 576-82.

35. Pauli C, Fintelmann R, Klemens C, et al. [Polyposis nasi-improvement in quality of life by the influence of leukotrien receptor antagonists]. Laryngorhinootologie 2007; 86: 282-6.

36. Bavbek S, Celik G, Ozer F, Mungan D, and MISIRLIGIL Z. Safety of selective COX-2 inhibitors in aspirin/nonsteroidal anti-inflammatory drugintolerant patients: comparison of nimesulide, meloxicam, and rofecoxib. J Asthma 2004; 41: 67-75.

37. Knowles SR, Drucker AM, Weber EA, and Shear $\mathrm{NH}$. Management options for patients with aspirin and nonsteroidal antiinflammatory drug sensitivity. Ann Pharmacother 2007; 41: 1191200.

38. Martin-García C, Hinojosa M, Berges P, et al. Safety of a cyclooxygenase-2 inhibitor in patients with aspirin-sensitive asthma. Chest 2002; 121: 1812-7.

39. Celiker V and Basgul E. Anaesthesia in aspirininduced asthma. Allergol Immunopathol (Madr) 2003; 31: 338-41.

40. Batra PS, Kern RC, Tripathi A, et al. Outcome analysis of endoscopic sinus surgery in patients with nasal polyps and asthma. Laryngoscope 2003; 113: 1703-6.

41. Robinson JL, Griest S, James KE, and Smith TL. Impact of aspirin intolerance on outcomes of sinus surgery. Laryngoscope 2007; 117: 82530.

42. Loehrl ta, Ferre RM, Toohill RJ, and Smith TL. Long-term asthma outcomes after endoscopic sinus surgery in aspirin triad patients. $A m \mathrm{~J}$ Otolaryngol 2006; 27: 154-60.

43. Pfaar 0 and Klimek L. Aspirin desensitization in aspirin intolerance: update on current standards and recent improvements. Curr Opin Allergy Clin Immunol 2006; 6: 161-6.

44. Stevenson DD and Simon RA. Selection of patients for aspirin desensitization treatment. $J$ Allergy Clin Immunol 2006; 118: 801-4.

45. Lee JY, Simon RA, and Stevenson DD. Selection of aspirin dosages for aspirin desensitization treatment in patients with aspirin-exacerbated respiratory disease. J Allergy Clin Immunol 2007; 119: 157-64.

46. Silberman S, Neukirch-Stoop C, and Steg PG. Rapid desensitization procedure for patients with aspirin hypersensitivity undergoing coronary stenting. Am J Cardiol 2005; 95: 50910.

47. Rossini R, Angiolillo DJ, Musumeci G, et al. Aspirin desensitization in patients undergoing percutaneous coronary interventions with stent implantation. Am J Cardiol 2008; 101: 786-9.

48. PariKh AA and Scadding GK. Intranasal lysineaspirin in aspirin-sensitive nasal polyposis: a controlled trial. Laryngoscope 2005; 115: 1385-90.

49. Lee RU, Simon RA, and Stevenson DD. Intranasal Ketorolac for Desensitization in Patients with Aspirin Exacerbated Respiratory Disease. J Allergy Clin Immunol 2008; 121: 1.

50. Macy E, Bernstein Ja, Castells MC, et al. Aspirin challenge and desensitization for aspirinexacerbated respiratory disease: a practice paper. Ann Allergy Asthma Immunol 2007; 98: $172-4$ 